\title{
Propuesta de factores clave de éxito para la implementación de ambientes virtuales de aprendizaje en educación superior
}

\author{
Armando Sánchez Macías*, María Guadalupe Veytia Bucheli**
}

\begin{abstract}
RESUMEN
La propuesta Factores Clave de Éxito para la implementación de Ambientes Virtuales de Aprendizaje en Educación Superior es el resultado de un proceso de reflexión y argumentación desde una perspectiva teórico-empírica, con el fin de profundizar en relación a dos términos clave: entorno y ambiente. Ambos conceptos son analizados epistemológica y conceptualmente desde diferentes contextos y perspectivas a objeto de evidenciar sus rasgos y características propias y valorar su empleo en el ámbito educativo a partir del manejo de las TIC en la educación terciaria.
\end{abstract}

Palabras clave: Factores clave de éxito, ambientes virtuales de aprendizaje, Educación Superior.

Proposed Key factors for success when implementing virtual learning environments in Higher Education

\section{ABSTRACT}

The proposal Key Success Factors for the implementation of Virtual Learning Environments in Higher Education is the result of a process of reflection and argumentation from an empirical-theoretical perspective with the end to deepen the two key terms relationship environment and atmosphere in virtual learning. Both concepts were analyze epistemologically and theoretically from different contexts and perspectives to show their own features and characteristics and asses its employment in the educational field trough the management of the TIC in the tearthiary education.

Keywords: Critical success factors, virtual learning athmosphere, higher education

* Armando Sánchez Macías es Doctor en Educación y Académico de la Universidad Autónoma de San Luis Potosí. E-mail: asanchezm76@hotmail.com

María Guadalupe Veytia Bucheli es Doctora en Gestión Educativa y Académica de la Universidad Autónoma del Estado de Hidalgo. E-mail: dra.veytiabucheli@gmail.com 


\section{Introducción}

La Sociedad del Siglo XXI se caracteriza por el fenómeno de globalización, los cambios que se generan en los diferentes ámbitos económicos, sociales, culturales, educativos; lo cual se ha visto influenciado por el auge de las Tecnologías de la Información y la Comunicación (TIC). En este sentido, la UNESCO (2005, p. 29) asegura que es imprescindible fortalecer "la capacidad para identificar, producir, tratar, difundir y utilizar información con vistas a crear y aplicar los conocimientos necesarios para el desarrollo del ser humano". Este proceso se ha complejizado a partir del empleo de las TIC, por la cantidad de información que se genera en diferentes tipos y modalidades.

Al abordar la importancia de obtener información para construir conocimiento, Castells (1999) asegura que el empleo de las TIC ha propiciado nuevas maneras de relación para llevar a cabo estos procesos, donde las distancias se acortan y se construyen diversas formas de comunicación y de relación entre los sujetos, tanto de manera sincrónica como asincrónica, lo cual permite eliminar las barreras del tiempo y del espacio, además de que se destacan como rasgos de la sociedad actual la flexibilidad, la adaptabilidad y la capacidad de supervivencia ante los cambios que se presentan de forma continua, desde esta dinámica, uno de los conceptos clave que comienza a cobrar fuerza es el de sociedad en red, siendo la colaboración una columna vertebral para la construcción de conocimientos.

El empleo de las TIC en los diferentes espacios de la vida del ser humano es cada vez más frecuente y, por supuesto, el ámbito educativo no es la excepción, ya que los Procesos de Enseñanza Aprendizaje (PEA) se enriquecen con el manejo de diferentes herramientas que favorecen la construcción del conocimiento, tanto de forma individual como colaborativa, a partir de trabajar en las modalidades presenciales, semipresenciales y abiertas en sus diferentes niveles educativos.

La incorporación de las TIC en el espacio educativo se ha llevado considerando un proceso paulatino. De acuerdo a Moreno (2010) es 
posible identificar tres formas en las cuales se han hecho presentes en educación: la primera de manera cuantitativa, si se observa únicamente desde el aspecto de la infraestructura y la accesibilidad; la segunda desde una perspectiva cualitativa, en donde se destaca la necesidad de incorporar las TIC en el PEA y, por último, la tercera como una herramienta que favorece la indagación y la investigación sobre el empleo de las interacciones que se llevan a cabo entre los sujetos y el proceso de apropiación de las TIC, tanto de docentes como de alumnos.

En relación a este punto, la UNESCO (2008) asegura que el nivel de apropiación tecnológica en el ámbito educativo se analiza desde tres enfoques principalmente: el primero aborda las nociones básicas de TIC, donde se incorporan los planes de estudio y, de esta manera, los docentes y estudiantes comienzan a emplearlas en el PEA. El segundo enfoque se refiere a la profundización del conocimiento, presentando un impacto mayor, ya que a partir del empleo de las TIC se pretende enriquecer el análisis de determinadas problemáticas tanto de forma individual como de manera colaborativa y, con base en ello, generar acciones que permitan dar solución a las situaciones identificadas. Finalmente, el tercer enfoque es el de generar conocimiento en el cual se busca una participación activa de docentes y estudiantes a través de acciones caracterizadas por la creatividad, la innovación y el pensamiento crítico.

A continuación, se lleva a cabo un análisis orientado a conceptualizar los Ambientes Virtuales de Aprendizaje (AVA), los Entornos Virtuales de Aprendizaje (EVA) y, a partir de esto, identificar los factores que contribuyen al desarrollo de los AVA y los EVA en la Educación Superior.

\section{Ambientes Virtuales de Aprendizaje}

Del latín ambiens, -entis "que envuelve o cerca" la palabra ambiente para la Real Academia Española (2014a, s/p) es lo que "rodea algo o a alguien como elemento de su entorno". En el contexto educativo un ambiente de clase es el "marco físico, psicológico y social en el que se educan los alumnos” (Martínez-Otero, 2000, p. I37); es un 
"sistema dinámico, que incluye tanto la conducta docente como la interacción profesor/alumno y la interacción entre alumnos” (Gómez, Valle y Pulido, 1989, p. 368).

El ambiente implica la interacción del ser humano con el entorno natural y social que lo rodea. La concientización del ser humano que lo traslada del soporte hacia el mundo, y de la vida hacia la existencia, la cual implica "(...) necesariamente el lenguaje, la cultura, la comunicación en niveles más profundos que lo que ocurría y ocurre en el dominio de la vida" (Freire, 2006, p. 5I). El ambiente de aprendizaje requiere de esta concepción activa, consciente del ser humano, por ende, incluye acciones pedagógicas en las que el que aprehende puede reflexionar sobre sus acciones y las de los demás en relación con el ambiente (Duarte, 2003).

El ambiente ha sido, también, definido como "el conjunto de factores internos -biológicos y químicos-y externos -físicos y psicosocialesque favorecen o dificultan la interacción social" (Sauve, I994, p. 2I). En este sentido, Sauve (1994) propone dimensiones superpuestas en las que se desenvuelve el ser humano y percibe al ambiente; esto desde varias perspectivas que son incluyentes y complementarias y que reunidas conforman la cosmovisión propia de cada pueblo. Así, el medio ambiente es la fuente de problemas a investigar; el recurso primero para ser gestionado con responsabilidad; la naturaleza de la que procedemos; la biosfera que se comparte y cohabita con los demás seres. Por su parte, Motta (1995) argumenta que la combinación de estas percepciones produce culturas que asumen a la naturaleza como dadora de vida, mientras que para otras es un objeto de explotación. El ser humano se relaciona con el ambiente a través de un proceso histórico cultural, a veces, adaptándose y, a veces, transformándose mediante una correlación simbólica en la que se interpreta permanentemente la realidad (Díaz y Gómez, 2005).

Los autores de este trabajo asumen al ambiente como algo más que un espacio físico que rodea y envuelve; es el espacio para la interacción del ser humano histórico con su contexto cultural, creándolo y transformándolo, a su vez, el ambiente ejerce su influencia en el sujeto, siempre en un ciclo dialéctico. De esta manera, el ambiente es el lugar en el que se encuentran los problemas que el ser humano 
debe resolver en su vida laboral y en sus otros roles y dimensiones. Por lo tanto, el docente debe concebirlo como la fuente y origen de las experiencias de aprendizaje que ha de tomar y, luego, diseñar mediante la didáctica para lograr el desarrollo de sus alumnos. Es en el ambiente en el que el alumno observa, convive, investiga, experimenta, infiere y siente, en consecuencia, es el auténtico espacio significativo para el aprendizaje. Si el espacio se refiere al lugar físico donde ocurren los procesos humanos, luego el ambiente es el espacio interpretado y concebido por la persona, agente transformador y transformado.

El ambiente comúnmente se define acompañado del concepto aprendizaje como una "concepción activa que involucra al ser humano y, por tanto, involucra acciones pedagógicas en las que quienes aprenden están en condiciones de reflexionar sobre su propia acción y sobre las de otros, en relación con el ambiente" (Raichvarg en Duarte, 2003, p. 2). Para Naranjo y Torres (1996) y Pergolis (2000) el ambiente de aprendizaje es la interacción dinámica de la "ciudad educativa" con el ser humano que actúa en el proceso educativo, por lo cual es indispensable tomar en cuenta todos los elementos intrínsecos y extrínsecos del lugar y de los sujetos del proceso enseñanza aprendizaje.

Para Ospina (1999) el ambiente de aprendizaje implica un trabajo de edificación permanente, que nos lleva a repensar permanentemente en ese espacio que permita la diversidad para propiciar el intercambio de la riqueza del individuo con lo que lo rodea. Finalmente, Cano y Lledó (1995) proponen una serie de principios indispensables para poder crear ambientes de aprendizaje apropiados, que implican, a saber: a) establecer las condiciones para la cohesión del grupo en torno a objetivos comunes, b) el entorno como medio diverso que permita la interacción con diferentes actividades que propicien aprendizajes afectivos, sociales y cognitivos; c) ofrecer escenarios diversos para estimular el aprendizaje; d) ofrecer, a su vez, subescenarios que permitan a las personas sentirse admitidas, considerando sus propias

I Proceso de compenetración íntima de la educación y del tejido, social, político y económico, en las células familiares para poner a la libre disposición de cada ciudadano los medios de instruirse, formarse, cultivarse a su propia conveniencia, de modo que conciba su educación como su responsabilidad y no como su obligación (Faure et al., 1973). 
características, y e) la identidad del ambiente de aprendizaje debe ser el reflejo de la identidad del grupo.

Como se infiere, el ambiente de aprendizaje considera una dimensión física que enlaza infraestructura educativa y la disposición espacial del lugar en el que se desarrolla el proceso de enseñanza aprendizaje. Sin embargo, la dimensión social está presente y es muy importante, por tanto, es necesario relacionar las acciones individuales e interactivas, las experiencias previas y las que se van desarrollando entre los participantes, todas ellas condicionadas y determinadas por su contexto histórico cultural. En palabras de Chaparro (I995, p. 2) el ambiente físico incorpora:

(...) Las dinámicas que constituyen los procesos educativos y que involucran acciones, experiencias y vivencias por cada uno de los participantes; actitudes, condiciones materiales y socioafectivas, múltiples relaciones con el entorno y la infraestructura necesaria para la concreción de los propósitos culturales que se hacen explícitos en toda propuesta educativa.

Ahora bien, los procesos educativos basados en plataformas informáticas, es decir, en modalidad no escolarizada y que son llamados, también, no presenciales o educación a distancia, añaden el concepto de lo virtual, que "tiene existencia aparente y no real", según la Real Academia Española (20I4b, s/p). En estos la mediación digital se considera imprescindible y en México la autoridad educativa los denomina Educación Virtual, definiéndolos como aquellos en los que:

(...) No existen necesariamente coincidencias espaciales y/o temporales entre quienes participan en un programa académico y la institución que lo ofrece. Esta circunstancia implica estrategias educativas y tecnológicas específicas para efectos de comunicación educativa, acceso al conocimiento, procesos de aprendizaje, evaluación y gestiones institucionales (Secretaría de Educación Pública, 2008, p. 5).

Es así como se llega al concepto de Ambiente Virtual de Aprendizaje (AVA) que viene a colocar en espacios no físicos las relaciones del ambiente con los sujetos involucrados en el Proceso Enseñanza 
Aprendizaje (PEA). Los elementos de los AVA incluyen a los usuarios (estudiantes y asesores), los contenidos, los especialistas (que diseñan, desarrollan y materializan los contenidos en objetos de aprendizaje $e^{2}$ y el Sistema de Administración del Aprendizaje (LMS, por sus siglas en inglés). Considerando a sus elementos, el AVA es el "(...) conjunto de entornos de interacción, sincrónica y asincrónica, donde, con base en un programa curricular, se lleva a cabo el proceso enseñanza aprendizaje, a través de un sistema de administración de aprendizaje” (López, Escalera y Ledesma, 2002, p. 9).

Haciendo una recapitulación acerca del concepto de AVA se asume, entonces, como:

- Un lugar sistemático, organizado pero maleable y adaptativo, que permite el desarrollo de la heurística y la aplicación de las habilidades metacognitivas, actitudes y emociones. Un lugar que propicie la transferencia del aprendizaje en el alumno (Mayer, 2000).

- Un espacio que favorezca el aprendizaje autónomo como capacidad para hacerse cargo del propio aprendizaje (Holec, I98I) con sus particularidades, un proceso autorregulado, intencional, acumulativo y constructivo. Que fomente la interacción para la colaboración y cooperación de los alumnos, así como el desarrollo de valores.

- Un modelo de aprendizaje activo que permita construir conocimientos significativos de preferencia basado en experiencias reales de los alumnos (Ausubel, 1976).

- Un sitio que propicie la exaltación del ser humano mediante el desarrollo de su "(...) capacidad transformadora, innovadora, emprendedora y creadora (...) que los comprometa con la sociedad en que viven (...) para alcanzar el máximo desarrollo personal que contribuya (...) al pleno desarrollo de la sociedad y la humanidad en su conjunto" (Zilberstein y Olmedo, 2016, p. 67).

2 Los objetos de aprendizaje son unidades de contenido digital que tienen un propósito educativo y que pueden ser utilizados en diversos contextos (Serrano, 2010). 
Como ya se presenta arriba, los AVA incluyen sustancialmente entornos de aprendizaje, los cuales se revisarán a continuación.

\section{Entornos Virtuales de Aprendizaje}

Entorno quiere decir ambiente o "lo que rodea". En el ámbito de la Informática es un "conjunto de características que definen el lugar y la forma de ejecución de una aplicación” (Real Academia Española, 20I4, s/p). A pesar de que puede utilizarse como un sinónimo de ambiente, cuando se refiere a las ciencias pedagógicas cada uno de estos conceptos se identifica con significados distintos, o si se prefiere, complementarios.

En la actualidad, se transita en la época en que las relaciones persona a persona coexisten con las relaciones basadas en la virtualidad, esto gracias a las bondades de las TIC. En estos ambientes, los individuos, las organizaciones, las identidades y los roles se han transformado, dando como resultado nuevos medios para la producción de la cultura y socialización mediatizada. En este sentido, Martín-Barbero (2002, p. 8I) se refiere a la cibercultura como un "universo mediáticorelacional, el espacio de los lenguajes y el tiempo de las nuevas comunicaciones".

Se convierte en una necesidad el identificar y describir cómo se ve influenciado el PEA en la sociedad del conocimiento, en los que además del lenguaje oral y escrito, el icónico adquiere gran importancia por las implicaciones que tiene en el pensamiento visual y porque es una de las formas más importantes para comunicarse en los Entornos Virtuales de Aprendizaje (EVA). Vale la pena resaltar que la estructuración de contenidos con fundamentos pedagógicos y didácticos propios del entorno virtual da lugar a la posibilidad de educar hacia objetivos preestablecidos. Se debe considerar evidentemente que los roles del estudiante, del profesor, tutor o facilitador cambian, y que el diseño pedagógico debe estar en sintonía con estas exigencias. Más aún es importante identificar si los actores son nativos o inmigrantes digitales que deben adaptarse al nuevo entorno o ambiente (Prensky, 200I); o en su caso, las consideraciones si es que los usuarios son residentes, acostumbrados e identificados con el 
uso de herramientas en Internet, o visitantes, los cuales son escépticos de expresarse mediante la cultura en línea (White y Le Cornu, 20II).

Es indispensable considerar que este nuevo contexto trastoca las condiciones originales en que se imparte la educación. De manera estructurada y gradual, la planeación de la escuela presencial ha sido sacudida por la televisión y el Internet que le permiten al individuo, ya sea niño o adulto, recibir una gran cantidad de información de manera sencilla y rápida. Para Martínez-Barbero (1996) este saber visual produce un desorden cultural que deslegitima y deslocaliza la autoridad del maestro y de la escuela. Dicho sea de paso los contenidos a los que se accede en su mayoría no son de la mejor calidad y mucho menos atienden a los propósitos educativos.

Así pues, el Entorno Virtual de Aprendizaje (EVA) se entiende como la organización de exigencias y procesos para gestionar un curso de tal forma que permita facilitar el aprendizaje por parte de los participantes. Para lo cual el diseño deja su forma lineal-secuencial para convertirse en uno dialéctico en forma de espiral constructivo, el cual implica la conciencia sobre el hecho educativo y las actividades propias, una permanente evaluación y una desestructuración secuencial rígida para darle lugar a formas flexibles donde los procesos creativos tengan cabida (Quiñones, 2006). Dicha organización va más allá de los elementos tecnológicos y de las representaciones culturales, la interacción entre los participantes, lo económico y lo político (Zambrano, 2000).

En el Entorno Virtual de Aprendizaje (EVA) la posibilidad de interactuar con el medio establece condiciones más naturales para las nuevas generaciones. Se puede elegir el orden de la información que se va a estudiar, la velocidad, y el tiempo que se va a dedicar, además se puede escoger la forma como se quiere relacionar con el hecho educativo en los AVA.

Los principios y regularidades le dan sentido a una ciencia. En el caso de la Pedagogía, es necesario revisar desde las corrientes educativas cómo adaptarse a los contextos virtuales. Se considera factible en particular desde el constructivismo, considerando que en esta teoría educativa se asume al medio ambiente como un elemento fundamental 
en el proceso de desarrollo del alumno. El sujeto es el resultado de las interacciones de las disposiciones internas y el medio ambiente, por ende, la construcción del ser humano está en relación con su medio (Carretero, 1997).

Los principios pedagógicos que deben ser considerados en el desarrollo y gestión de Entornos Virtuales de Aprendizaje (EVA) incluyen, a saber (Quiñones, 2006):

- Un apropiado balance entre el conocimiento y la comunicación, poniendo énfasis en la relación y no en la cantidad. Dado que no se pretende la transmisión de conocimiento, se requiere que el diseño de los objetos de aprendizaje estén desarrollados bajo procesos de apropiación social y cultural que lleven a aprendizajes significativos.

- La tecnología como medio para el diseño de nuevos dispositivos pedagógicos que permitan la construcción de competencias en ambientes colaborativos, utilizando estructuras no lineales, asociativas y jerárquicas, incorporando diversidad de medios y la comunicación síncronica y asíncronica.

- El uso de objetos de aprendizaje con problemas del mundo real que le otorguen al estudiante control sobre su proceso de instrucción para que a través del análisis-síntesis, la induccióndeducción, la abstracción-concreción, la modelación y lo histórico-lógico descubra el sentido de sus experiencias y, a la vez, construya su propio conocimiento.

- La utilización de estrategias de apoyo cognitivo, tales como la tutoría, el modelamiento y el aprendizaje guiado en el que la plataforma educativa sirve como medio para que el estudiante construya con libertad y flexibilidad representaciones de conocimiento propias. Como contraposición al uso esquemático, estático y secuencial propio de las estructuras tradicionales de la enseñanza.

Como ya se definió antes, los entornos forman parte de los ambientes, López, Escalera y Ledesma (2002) enuncian aquellos EVA que operan en un AVA: 
- El entorno de conocimiento que se deriva del diseño curricular y se refiere a los contenidos que, a su vez, se presentan como objetos de aprendizaje que como característica deben ser interactivos y adaptados al medio;

- El entorno de colaboración en el cual se da la interacción entre los actores, también llamada realimentación, puede ser sincrónica o asincrónica;

- El entorno de asesoría en el cual se presta la tutoría del facilitador hacia el alumno de manera personalizada y que, generalmente, es asincrónica;

- El entorno de experimentación que sirve como complemento a los contenidos para darle carácter vivencial si es pertinente con los contenidos, finalmente;

- El entorno de gestión mediante el cual el asesor controla el proceso pedagógico y escolar, así como los alumnos interactúan con este.

A continuación, se presenta la concepción de los AVA de los autores desde el enfoque de sistemas (Bertalanffy, 2006), pues como sistema se encuentra subordinado a un suprasistema que es el medio ambiente y, a su vez, está constituido por componentes que son sus infrasistemas, que cumplen con las características de asumir funciones específicas dentro de una estructura integrada de forma lógica-secuencial y que apuntan a la consecución de objetivos previamente establecidos.

Los AVA se constituyen de elementos de dos tipos, que, al mismo tiempo, intervienen en diferentes momentos de una secuencia sistémica-cíclica, a saber:

- Objetos: los objetos de aprendizaje, las TIC y LMS, los contenidos, la gestión, las actitudes, la autonomía, la autorregulación, el pensamiento crítico e innovador, la construcción de conocimientos, entre otros.

- Sujetos: los especialistas (pedagogos, diseñadores, expertos en los contenidos y en la gestión de las TIC), los alumnos que son los destinatarios del PEA, los asesores (facilitadores o docentes) y la sociedad dado que a final de cuentas la educación la tiene como último y principal destinatario de sus esfuerzos. 
Cabe destacar que estos elementos interrelacionados se encuentran en un ambiente multidimensional generado por los diferentes EVA (Ver Figura I).

Figura I. AVA desde el enfoque de sistemas

MEDIO AMBIENTE

MEDIO AMBIENTE

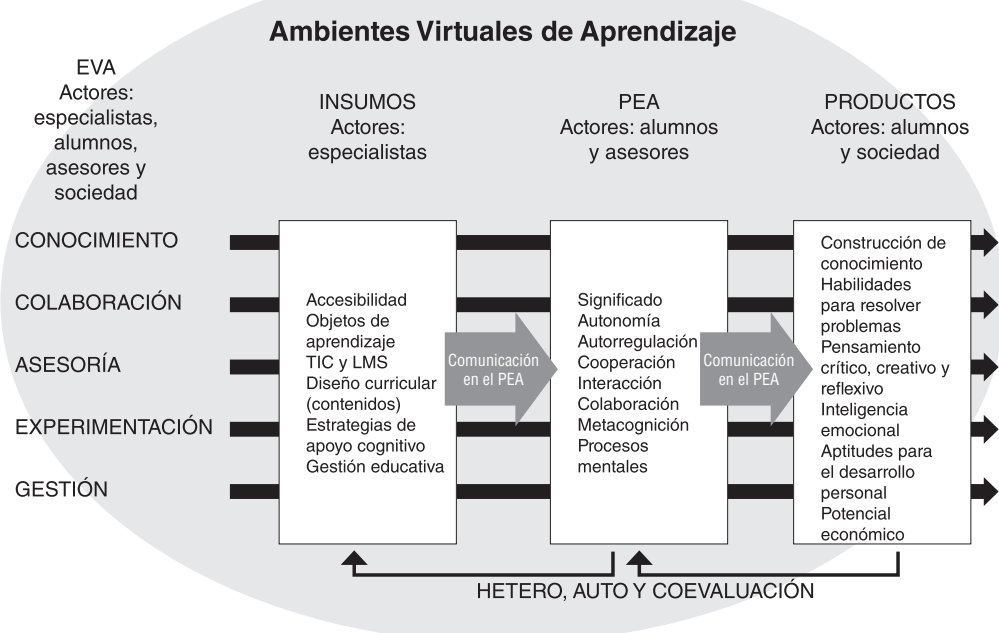

MEDIO AMBIENTE

MEDIO AMBIENTE

Fuente: Elaboración propia.

Características de los AVA y los EVA en algunas de las universidades virtuales mexicanas

Bustos y Coll (2010) afirman que la incorporación de las TIC en el PEA se puede observar desde diferentes niveles:

a) Incorporación de las TIC como un complemento del PEA;

b) Como manejo de contenidos específicos;

c) Herramientas para apoyar en un contenido de manera específica;

d) Se distribuyen a los estudiantes gracias a su portabilidad, y,

e) La conexión se lleva a cabo en red.

Llevar a cabo un análisis del empleo de las TIC en la Educación Superior refleja el incremento significativo que se ha tenido en los últimos años, sin embargo, este proceso ha representado un camino complejo y paulatino, presentando cierta resistencia por parte, tanto 
de docentes como de estudiantes para incorporarlas en los PEA. Díaz Barriga (2013) asegura que el proceso de incorporación se puede clasificar desde tres tipos de tendencias: la primera, en la cual se busca habilitar al docente en el empleo de las TIC; la segunda, que se orienta hacia el manejo de los contenidos que puedan ser utilizados por los docentes y los alumnos en línea, y la tercera, donde se aborda el empleo de las TIC no únicamente desde una dimensión tecnológica, sino desde una perspectiva más integral a partir de un sustento psico-pedagógico. En este caso, se consideran las características de los estudiantes, como puede ser su edad, sus intereses y, con base en ellas, se diseñan las secuencias didácticas para construir el aprendizaje, tanto de manera individual como de forma colaborativa, recuperando los saberes previos de cada uno de los integrantes y, de esta manera, transitar de islas de conocimientos a redes de conocimientos.

Lo relevante es trabajar las TIC no solo como una herramienta tecnológica, sino como una herramienta que vincule la tecnología y la didáctica para incorporarlos a los procesos de enseñanzaaprendizaje. En este sentido, Silvio (2004) asegura la importancia de transitar de un paradigma orientado a la enseñanza y transmisión de conocimientos a uno orientado al aprendizaje desde la construcción de conocimientos, en donde el rol del docente sea un guía, un facilitador del aprendizaje a partir de la generación de Comunidades Virtuales de Aprendizaje (CVA) que se caracterizan por relaciones de horizontalidad, tanto de manera sincrónica como asincrónica y en las cuales se favorece la construcción de redes de conocimiento entre compañeros y docentes.

Con base en los argumentos anteriores, Badía (2006) plantea que el manejo de las TIC por sí solo no genera prácticas constructivistas en el PEA. De esta forma, es fundamental tener una intencionalidad clara, así como llevar a cabo procesos de planeación adecuada en donde se consideren los siguientes componentes: los docentes desde una función de guía, mediadores y facilitadores; los estudiantes desde un rol activo en que sean los protagonistas de la construcción del conocimiento; los contenidos incluidos en los cursos y la manera en cómo están diseñados y, finalmente, el empleo de las TIC como 
articulador de los contenidos presentados con los sujetos que son ya sea docentes como estudiantes.

Al respecto, De Souza (2007) afirma que el mercado educativo se ve marcado por la Sociedad de la Información y la economía basada en el conocimiento, en que el empleo de las TIC genera un nuevo tipo de gestión y es diferente la relación que se establece entre los sujetos, la información y el conocimiento, los que van generando diferentes redes de colaboración, nuevas pedagogías y nuevas maneras de difundir el conocimiento científico.

Estudios realizados por Ersad, Gilie y Arnseth (2013) aseguran que dentro de los principales retos a los que se enfrenta la Sociedad del Siglo XXI se encuentran los procesos que permitan comprender las interconexiones que se llevan a cabo entre los sujetos, las redes y los procesos de comunicación que se establecen, tanto de manera sincrónica como asincrónica a partir del empleo de las Tecnologías de la Información y la Comunicación, y la manera como la información se convierte en conocimiento a partir de su identificación, empleo, análisis y transferencia a otros contextos, donde es relevante el estudio que adquieren las trayectorias de los sujetos que emplean las TIC.

Cassany (2012), por su parte, destaca algunos cambios más significativos que se presentan en los procesos de enseñanzaaprendizaje actuales al utilizar las TIC. Principalmente, se ha incrementado la cantidad de información a la que se tiene acceso, la cual puede ser vista en diversos formatos, pero no existen filtros para valorar qué información es pertinente y cuál no. En este sentido, se ha diversificado la manera de escribir, los tiempos en los que se realiza la escritura y es más frecuente utilizar formas de trabajo colaborativas que individuales.

A su vez, Rodríguez (2015) comenta que los nuevos contextos han cambiado radicalmente, lo cual impacta, por cierto, el ámbito educativo y el concepto de aprendizaje se ha vuelto cambiante, no tiene un valor único, verdadero y definido, sino que se ha ido transformando, construyendo y enriqueciendo. Asimismo, la noción de contexto se ha diversificado, ya no solo se consideran los contextos 
presenciales, sino también los semipresenciales y los virtuales y con ello los procesos de enseñanza-aprendizaje que se construyen en cada uno de estos contextos.

Al revisar los planteamientos anteriores sobre la incorporación de las TIC en la sociedad en general y de manera particular en el ámbito educativo, es relevante precisar que las Instituciones de Educación Superior (IES) han generado cambios en su oferta educativa, lo cual les ha permitido dar respuesta a las demandas de la sociedad actual. Desde esta perspectiva, es que Rama (20I4) lleva a cabo un análisis de la incorporación de las TIC en las universidades, donde destaca que este fenómeno amplió la cobertura de la educación y se han incorporado a estos espacios personas que trabajan y están interesados en seguir estudiando, por lo que es frecuente observar en las IES ofertas de programas en modalidad semipresencial, la virtualización de programas presenciales, así como el diseño y desarrollo de programas en modalidad virtual.

En este proceso de reingeniería de la Educación Superior en América Latina, Rama (2014) asegura que la educación a distancia se inicia a finales de los años 70 a partir de la creación de modelos semipresenciales en países como México, Ecuador, Colombia, Venezuela y Costa Rica, destacándose universidades con esfuerzos muy importantes, entre ellas, la Universidad Abierta y a Distancia de México (UNAD), la Universidad Técnica Particular de Loja en Ecuador (UTPL), la Universidad Nacional de Costa Rica (UNA) y el Instituto Tecnológico de Estudios Superiores de Monterrey en México (ITESM).

Actualmente en México se encuentran varias instituciones que se crearon ya en la virtualidad como son: Universidad Virtual de la Universidad de Guadalajara (pública), Universidad Virtual del Estado de Guanajuato (pública), Consorcio Clavijero del Estado de Veracruz (pública), Universidad Virtual del Estado de Michoacán (pública), Universidad Mexicana en Línea (privada), Instituto Tecnológico de Monterrey Virtual (privada), Universidad Autónoma del Estado de Hidalgo en la modalidad virtual (pública), Universidad DaVinci (privada), Universidad Tecnológica Privada en Línea UTEL (privada). 
Dentro de las características que se presentan con mayor frecuencia en los modelos de las universidades virtuales mexicanas se encuentran los siguientes:

- Flexibilidad: el estudiante tiene la libertad de organizar su ritmo de estudio y el tiempo que dedica para la realización de sus actividades y tareas.

- Inclusión: pueden estudiar personas que les es complejo trasladarse de un lugar a otro.

- Manejo de tecnología de vanguardia: se utilizan regularmente plataformas educativas, por ejemplo, Moodle, Sakai y Blackboard.

- Accesibilidad: los estudiantes ingresan a la plataforma para realizar sus actividades de acuerdo a sus tiempos, pues la mayoría de estas se lleva a cabo de manera asincrónica.

- Interactividad: en este tipo de programas se pueden llevar a cabo actividades tanto de manera sincrónica con el manejo de chat, como asincrónica con el empleo de las wikis y se realizan trabajos de forma individual y de manera colaborativa.

- Empleabilidad: los estudiantes fortalecen sus competencias profesionales y mejoran su desempeño en el ámbito laboral a partir de los contenidos y de las actividades que se les proponen.

En resumen, en los modelos virtuales los estudiantes, primero, desarrollan habilidades y actitudes que son buscadas y altamente valoradas por los empleadores en las organizaciones y, segundo, se les orienta hacia el desarrollo de emprendimientos en diferentes contextos, a partir de la movilización de los propios saberes conceptuales, procedimentales y actitudinales.

\section{Factores de éxito para la implementación de los AVA}

Los AVA son sistemas que cumplen con objetivos pedagógicos que impulsan, a su vez, objetivos más grandes de las IES (en Administración se les llaman visión y misión). No cabe duda que se convierte en una actividad indispensable medir el rendimiento y 
el costo-beneficio de los recursos invertidos en los sistemas AVA (Sánchez, 2015). Dado el enfoque sistémico, la evaluación debe darse en la amplitud de las diversas direcciones. Todos los elementos deben estar sujetos a mediciones para poder corregir errores y para entender los aciertos, repetirlos y entrar en el ciclo virtuoso de la mejora continua. Dada la complejidad de estos sistemas es insuficiente concentrar la medición en el desempeño académico o en los niveles de deserción de los cursos en AVA. La medición debe ser global.

La medición de la calidad de la educación en línea ha sido ya abordada y existen diversos modelos para este propósito. En el análisis de Veytia y Rendón (20I4) se identifican las mejores prácticas recurrentes entre dichos modelos: el liderazgo institucional, la gestión del PEA, el apoyo al estudiante y al profesorado, el desarrollo de cursos, las TIC, la evaluación de capacidades y la efectividad en costos. En este sentido, refiriéndonos específicamente a la medición para la evaluación de los cursos en AVA se propone considerar las cuatro perspectivas procesales que son más importantes y decisivas para alcanzar objetivos según Kaplan y Norton (2014). Es importante aclarar que dichas perspectivas se presentan en una secuencia de importancia para la gestión, es decir, para que se dé lo segundo hay que atender lo primero y así sucesivamente (Ver Figura 2).

Figura 3. Perspectivas para medir efectividad del AVA basado en BSC

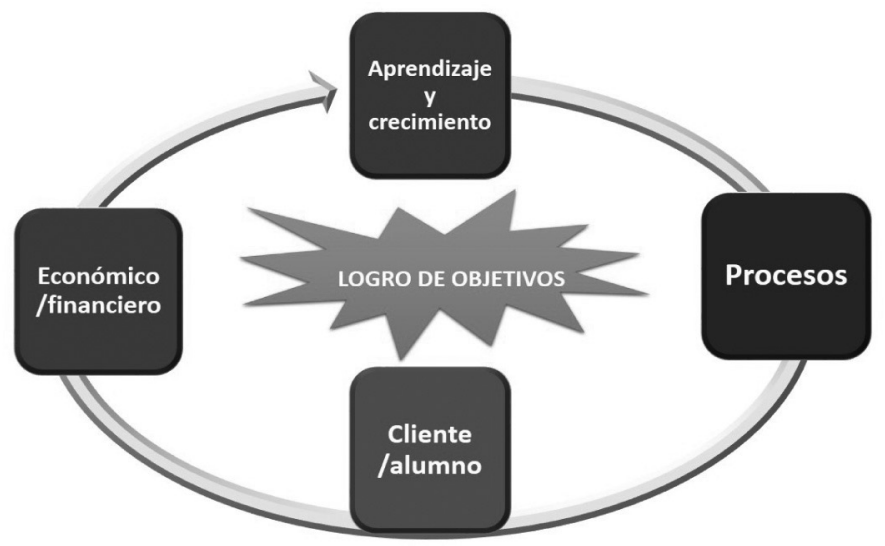

Fuente: Adaptado de Kaplan y Norton (2014). 
Se describen a continuación dichas perspectivas:

a) Perspectiva de aprendizaje y crecimiento: implica la gestión del capital humano y la gestión del conocimiento. Incluye la cultura organizacional, la capacitación y desarrollo del personal, la gerencia del conocimiento, los sistemas de memorización del aprendizaje organizacional. De esta perspectiva depende el éxito y la realización de las otras perspectivas. Responde a la pregunta: ¿en qué condiciones se encuentran las personas que ejecutarán los procesos?

b) Perspectiva en los procesos: se refiere a concentrar los esfuerzos en los procedimientos claves del sistema, vale decir, aquellos que agregan valor y son la fuente de ventajas competitivas sostenibles. Un procedimiento mal definido y estructurado nunca podrá ser ejecutado con calidad. El PEA sería por excelencia el proceso productivo que hay que detallar y pulir, pero también aquellos procesos de soporte que hacen posible que éste pueda llevarse a cabo.

c) Perspectiva en el cliente/alumno: se refiere a los clientes externos fundamentalmente, o sea, los alumnos. Una vez que se asegura que las personas que ejecutan los procesos están capacitados, los dominan, y su ejecución está adecuadamente estructurada, agregando valor y asegurando ventajas comparativas, lo siguiente es medir la satisfacción de nuestros clientes. La medición se basa en conocerlo y, luego, en contrastar las expectativas contra la percepción del servicio prestado.

d) Perspectiva económica/financiera: muy importante, pero únicamente realizable cuando las demás perspectivas se han logrado, es identificar qué tan efectivo ha sido el uso de los recursos y los beneficios que han generado. Esto no es exclusivo de las instituciones de educación privadas dado que las públicas, si bien no persiguen fines de lucro, sí están obligadas a prestar servicios a la mayor cantidad de usuarios con la mejor calidad posible dentro del presupuesto que tienen asignado. Todas las perspectivas anteriores tienen un impacto directo e irrevocable en esta.

Las ventajas del modelo son que traduce la visión y la estrategia de la institución educativa en indicadores medibles y cuantificables, permite comunicar y vincular los objetivos a acciones puntuales 
y da lugar a un aumento en la realimentación en el sistema. Para este propósito se debe generar un esquema de indicadores para cada perspectiva, los cuales son variables que informan acerca del funcionamiento o comportamiento de una actividad en la institución educativa. Los indicadores, también, son definidos como "(...) la relación entre variables cuantitativas y cualitativas que permiten observar la evolución o tendencias de cambio en el objeto o fenómeno observado respecto de objetivos y metas previstas e influencias esperadas" (Selltiz en Bermúdez y Rodríguez, 2013, p. 177).

Antes que indicadores, los autores prefieren llamarlos Factores Clave de Éxito (FCE) dado que son los elementos que identifican y hacen único al modelo que se implementa en la institución, es decir, debieran ser particulares y específicos en cada organización, asumiendo que se estructuran de manera innovadora y diferenciada para ser competitivas. Los FCE deben desprenderse del objetivo de la institución, expresarse a través de cifras que podrán aumentar o disminuir. Deben presentarse, además, de manera equilibrada para todas las perspectivas. Es muy importante que sean definidos por consenso entre los actores internos de la organización y considerar aquello a lo que le dan más valor los actores externos (clientes/ alumnos/sociedad). La cantidad de FCE depende de la suficiencia, no deben ser pocos que no midan lo necesario y no demasiados que desvirtúen la prioridad en la ejecución y la pongan, a su vez, en el control.

Es deseable y necesario que las IES entiendan su medio ambiente y en base a ello estructuren su modelo de aplicación de los AVA, considerando los elementos ya descritos. Una vez realizada esta tarea se considera indispensable identificar, describir y evaluar de manera continua los FCE con la finalidad de estudiar su evolución, proponer mejoras para incrementar la calidad de los servicios educativos y para predecir dificultades mediante el análisis de las tendencias.

\section{Conclusiones}

La colectividad se encuentra en una transformación constante. En la actual Sociedad del Conocimiento en la que los datos viajan 
y se intercambian incesantemente, es necesario que los procesos pedagógicos se adapten a las nuevas realidades y a las nuevas formas de pensamiento que esto ha desencadenado para darle sentido y propósito a estos cúmulos de información y se subordinen al bienestar del ser humano. El medio transforma al hombre y este, a su vez, al medio. Es por ello que la educación debe ser entendida en su más amplio sentido y debe considerar en su concepción pedagógica al ser humano como un ser histórico-cultural. El uso de las TIC con un modelo educativo apropiado es generador de grandes ventajas para las sociedades dado que con una cantidad menor de recursos puede llevarse la educación formal a donde antes era imposible. Se perfila el enfoque constructivista como el más apropiado para estos fines, pues su concepción teórica y práctica considera al medio ambiente como uno de los factores que fundamentalmente es parte natural del fenómeno de edificación y desarrollo del conocimiento en el sujeto.

Los autores consideran que es factible y viable el uso de las TIC con estos propósitos, además de que se consolida como una ventaja desde el punto de vista económico y que, desde luego, democratiza la educación. Dadas sus características, es relevante poner un enfoque especial en su adecuada implementación para disminuir la deserción de los cursos. Como se ha examinado, la creación de un EVA va mucho más allá que establecer objetivos, contenidos y tecnología. Los procesos tutoriales son fundamentales para la retención de la matrícula. Como cualquier proceso que se lleva a cabo en las organizaciones, debe ser estudiado y gestionado con un enfoque de sistemas dado que sus elementos están interrelacionados y son interdependientes. En consecuencia, se propone asumir desde las cuatro perspectivas a la organización, para a partir de ellas, identificar los Factores Clave de éxito (FCE) que desde las personas, los procesos, los alumnos y la dimensión económica den viabilidad y sustentabilidad a los programas educativos basados en AVA. En este sentido hay camino por recorrer.

\section{Referencias bibliográficas}

Ausubel, D. (1976). Psicología educativa: un punto de vista cognoscitivo. México: Trillas.

Bermúdez, L. T. \& Rodríguez, L. F. (2013). Investigación en la gestión empresarial. Bogotá: ECOE Ediciones. 
Bertalanffy, L. V. (2006). Teoría general de los sistemas: fundamentos, desarrollo, aplicaciones. México: Fondo de Cultura Económica.

Badía, A. (2006). Enseñanza y aprendizaje con TIC en la Educación Superior. Presentación. RUSC. Revista de Universidad y Sociedad del Conocimiento, 3(2), I-4.

Bustos, A. \& Coll, C. (2010) Los Entornos Virtuales como espacios de enseñanza y aprendizaje. Una perspectiva psicoeducativa para su caracterización y análisis. Revista Mexicana de Investigación Educativa, I5(44),I63-I84.

Cano, M. I. \& Lledó, A. (I995). Espacio, comunicación y aprendizaje. Sevilla: Diada.

Carretero, M. (1997). Constructivismo y educación. México: Progreso.

Castells, M. (I999). La sociedad red. México: Alianza Editorial.

Cassany, D. (2012). En_línea. Leer y escribir en la red. Barcelona: Editorial Anagrama.

Chaparro, C. I. (1995). El ambiente educativo: condiciones para una práctica educativa innovadora. Tunja: CINDE-UPTC.

De Souza, B. (2007). La Universidad en el Siglo XXI. Para una reforma democrática y emancipadora de la Universidad. Bolivia: CIDES-UMSA.

Díaz, R. \& Gómez, M. (2005). Representaciones del ambiente y organización social del espacio. Caso: Comunidad del sector "Chino Julio" del Municipio Maracaibo del Estado Zulia, Venezuela. Espacio Abierto, I4(4), 609-629.

Díaz Barriga, A. (2013). TIC en el trabajo del aula. Impacto en la planeación didáctica. Revista Iberoamericana de Educación Superior, 4(I0), 3-2I.

Duarte, J. (2003). Ambientes de aprendizaje. Una aproximación conceptual. Estudios Pedagógicos, 29, 97-II3.

Faure, E., Herrera, F., Kaddoura, A.-R., Lopes, H., Petrovski, A., Rahnema, M. \& Ward, F. (1973). Aprender a ser. La educación del futuro. París: UNESCO.

Freire, P. (2006). Pedagogía de la antonomía. Saberes necesarios para la práctica educativa. México: Siglo XXI editores.

Gómez, D., Valle, A. \& Pulido, M. (I989). Evaluación y estructura del clima socioescolar en alumnos de EE.MM. de Galicia. Revista de Psicología General y Aplicada, 92(3), 367-376.

Holec, H. (I98I). Autonomy and foreign language learning. Oxford: Oxford University Press.

Kaplan, R. S. \& Norton, D. P. (20I4). El cuadro de mando integral. Madrid: Planeta. 
López, A. E., Escalera, S. \& Ledesma, R. (2002). Ambientes virtuales de aprendizaje. Presimposio Virtual SOMECE 2002. México: SOMECE.

Martín-Barbero, J. (2002). La educación desde la comunicación. Bogotá: Norma.

Martínez-Otero, V. (2000). Formación integral de adolescentes: educación personalizada y programa de desarrollo personal (PDP). Madrid: Fundamentos.

Mayer, R. (2000). Diseño Educativo para un aprendizaje constructivista. En Reigeluth, C., Diseño de la instrucción. Teorías y modelos. Un paradigma de la teoría de la instrucción (pp.I53-I7I). Madrid: Siglo XXI.

Moreno, T. (2010). Competencias en educación. Una mirada crítica. Revista Mexicana de Investigación Educativa, I5(44), 289-297.

Motta, N. (1995). Reflexiones Antropológicas para un modelo de desarrollo alternativo y sostenible en la costa pacifica vallecaucana. Ponencia presentada en el III Congreso Latinoamericano de Ecología, Simposio Educación Ambiental para el Manejo de los Territorios. Mérida, Venezuela.

Naranjo, J. \& Torres, A. (I996). Ciudad educativa y pedagogías urbanas. Bogotá: Dimensión educativa.

Ospina, H. F. (1999). Educar, el desafío de boy: construyendo posibilidades y alternativas. Bogotá: Magisterio.

Pergolis, J. C. (2000). Relatos de ciudades posibles. Ciudad educadora y escuela, la práctica significante. Bogotá: Fundaurbana.

Prensky, M. (200I). Nativos o inmigrantes digitales. On the Horizon (MCB University Press, $9(5), \mathrm{I}-6$.

Quiñones, J. (2006). Fundamentos Pedagógicos en Entornos Virtuales de Aprendizaje. En Consejo Nacional de Acreditación, Entornos Virtuales en la Educación Superior (pp. 55-58). Bogotá: Consejo Nacional de Acreditación.

Real Academia Española (20I4a). Concepto de ambiente. Recuperado de http://dle.rae.es/?id=2HmTzTK

(20I4b). Concepto de virtual. Recuperado de http://dle.rae. es/?id=buDJhh3

Rama, C. (20I4). La virtualización universitaria en América Latina. Revista de Universidad y Sociedad del Conocimiento, II(3), 33-43.

Sánchez, A. (2015). Dimensión económica de la educación: eficacia y eficiencia. Educando para educar, I6(30), 63-76.

Sauve, L. (1994). Exploración de la diversidad de conceptos y de prácticas en la educación relativa al ambiente. Memorias Seminario Internacional la Dimensión Ambiental y la Escuela. Bogotá: Documentos Especiales MEN. 
Secretaría de Educación Pública (2008). Acuerdo número 445 por el que se conceptualizan y definen para la Educación Media Superior las opciones educativas en las diferentes modalidades. México: Autor.

Serrano, M. d. (2010). Objetos de Aprendizaje. Revista e-FORMADORES, I-5.

Silvio, J. (2004). Tendencias de la Educación Superior en América Latina y el Caribe. En IESALC, La Educación Superior virtual en América Latina y el Caribe (pp. 5-27). México: ANUIES.

UNESCO (2005). Hacia las sociedades del conocimiento. Francia: ONU.

UNESCO (2008). Estándares de competencia en TIC para docentes. Recuperado de: http://www.oei.es/tic/UNESCOEstandaresDocentes.pdf

Veytia, M. G. \& Rendón, F. (20I4). Medición de la calidad en la educación en línea: Un modelo sugerido en base a la perspectiva de los clientes. Ponencia presentada en el VII International GUIDE Conference 20I4 Cultural Identity in the Midst of Global Modernization: The Role of Distance Education. Ciudad de Guatemala, Guatemala.

Zambrano, M. F. (2000). El córtex digital: Posibilidades de desarrollo cognitivos en los nuevos espacios virtuales. En UPN, Relaciones y tensiones entre investigación e innovación en educación (pp. I4I-I74). Bogotá: Universidad Pedagógica Nacional.

Zilberstein, J. \& Olmedo, S. (2016). Didáctica desarrolladora, enfoque desde el enfoque histórico-cultural. Educaşão e Filosofia Uberlândia, 29(57), 6I-93. 